\title{
3D euclidean crystallography and hyperbolic orbifolds
}

Stephen Timothy Hyde ${ }^{1}$, Martin Cramer Pedersen ${ }^{2}$

${ }^{1}$ Research School Of Physics, Canberra, Australia, ${ }^{2}$ Niels Bohr Institute, Copenhagen, Denmark E-mail: stephen.hyde@anu.edu.au

Point and plane groups characterise isometries of the two-dimensional sphere and flat plane. Two-dimensional orbifolds afford a useful unifying concept to include also the third two-dimensional geometry: hyperbolic. For example, the same decoration of the $* 235, * 236$ and $* 237$ orbifolds gives the patterns shown in the figure: the first (reminiscent of C60) tiles the sphere, the second (graphene) tiles the plane and the third tiles the hyperbolic plane. The richness of hyperbolic orbifolds calls for some taxonomy. We have classified them into 7 classes, based on the orbifold topology [1]. Further, hyperbolic orbifolds fall into two categories: those that can be realised as crystallographic patterns in $3 \mathrm{~d}$ space and those that cannot. We can think of "crystallographic hyperbolic" orbifolds as sub-periodic groups, confined to periodic hyperbolic surfaces in $3 d$ space. These concepts are useful. They allow us to systematically enumerate nets of given (2d) topology. The natural hierarchy of orbifolds explains the prevalence of the genus-3 triply-periodic minimal surfaces in physical materials, from fluid membranes in vivo, to MOF structures.

[1] Hyde, S.T., Ramsden, S.J. and Robins, V., 2014. Unification and classification of two-dimensional crystalline patterns using orbifolds. Acta Crystallographica Section A: Foundations and Advances, 70(4), pp.319-337.

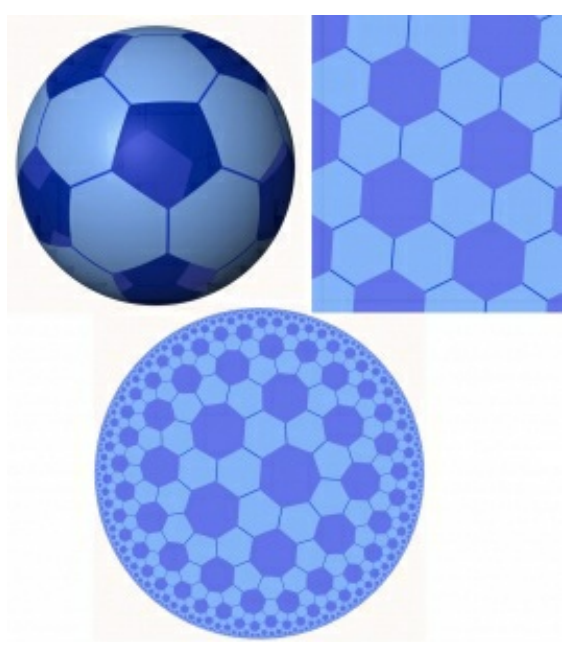

Keywords: hyperbolic crystallography, nets, graphs 